\title{
Lessons learned at the intersection of immunology and neuroscience
}

\author{
Lawrence Steinman
}

Neurology and Neurological Sciences, Stanford University School of Medicine, Stanford, California, USA.

\begin{abstract}
Neurobiologists and immunologists study concepts often signified with identical terminology. Scientists in both fields study a structure known as the synapse, and each group analyzes a subject called memory. Is this a quirk of human language, or are there real similarities between these two physiological systems? Not only are the linguistic concepts expressed in the words "synapse" and "memory" shared between the fields, but the actual molecules of physiologic importance in one system play parallel roles in the other: complement, the major histocompatibility molecules, and even "neuro"-transmitters all have major impacts on health and on disease in both the brain and the immune system. Not only are the same molecules found in diverse roles in each system, but we have learned that there is real "hard-wired" crosstalk between nerves and lymphoid organs. This issue of the JCI highlights some of the lessons learned from experts who are working at this scintillating intersection between immunology and neuroscience.
\end{abstract}

A few of us have the treasured opportunity to work at the interface of neuroscience and immunology. The nervous system and the immune system have distinctly different anatomic structures populated by very different cell types, yet the goals of each system have much in common. Both the nervous and immune systems are sensory: they sense changes in the environment and provide integrated responses that allow for survival. Just as the retina can be triggered by a few photons, the $\mathrm{T}$ cell receptor can signal with engagement of a single molecule and can form an immune synapse with engagement of as few as ten molecules (1). Both the nervous system and the immune system are also effectors: each system comprises efferent connections that masterfully orchestrate a response to what is sensed. Then there are the shared concepts between these two systems: we have neural synapses that integrate the strength of electrical signals between ensembles of neurons, allowing for processing of information from neural nets. Likewise, the immune system, using an architecture with some similarity to the neural synapse, integrates inputs from a variety of cell types with its own synapse on phagocytes, T cells, B cells, and NK cells.

The intersections between the brain and the immune system are even more astonishing: some of the classic neurotransmitters, such as the quintessential inhibitory molecule for neural synapses, GABA, also have an inhibitory function on immune synapses. And the most well-known molecules of the immune system are active within the nervous system: both the classical complement cascade and $\mathrm{MHC}$ molecules play stunning roles in axonal guidance and synaptic elimination (refs. 2, 3, and Figure 1).

These overlaps make "pathway analysis," a favorite tool of systems biologists and bioinformaticians, the target of a rude trick: molecules do not know whether they are in the "neurobiology club" or the "immunology club." Molecules have their own language: chemistry! We scientists who use pathway analysis describe molecules with words, but molecules are the essence of chemistry and our language can mask their actual roles. The correct language for neurobiology and immunology is not English or French or Spanish or Russian. The operative language is chem-

Conflict of interest: The author has declared that no conflict of interest exists. Citation for this article: JClin Invest. 2012;122(4):1146-1148. doi:10.1172/JCI63493. istry. So although we tend to think that complement and the MHC molecules are immunological, we should remember that this is a historical illusion that was shaped because we learned about their roles in immunity first, before we realized that they also play a remarkable part in the nervous system $(2,3)$. The illusion arose because we think in language and live creating our histories, while molecules engage in chemistry using a language of their own and without a history as we humans know it. The chemistry for both MHC and complement is congruent in the immune system and the nervous system, and this is true for a wide range of other molecules.

\section{Neurological and immunological synapses}

In the first review in this series, Michael Dustin describes the integration of multiple stimulatory and inhibitory signals (4), which is a feature of synapses in the nervous and immune systems. In the immune synapse, submicron-sized microclusters transmit these signals in a manner similar to that of the neural synapse. Also present in both synapse types are N-ethylmaleimide-sensitive factor attachment protein receptor (SNARE) proteins that are involved in the delivery of vesicles (5). As in the neural synapse, this process in the $\mathrm{T}$ cell synapse is sensitive to one of the quintessential neurotoxins, tetanus toxin (6). Here too, we observe that not only do immune and neural synapses have much in common in their functional design, but that there is a striking confluence of molecules involved in the physiology of both systems. SNAREs, neurotransmitters, and MHC molecules modulate formation and function of neurological and immune synapses. The well-known inhibitory transmitter of the nervous system, GABA, induces inhibitory cascades in T cells. Patch clamping of immune cells subjected to puffs and pulses of GABA delivered from a microelectrode reveals inward currents similar to what are seen in hippocampal neurons (7). Molecules of the class I MHC negatively regulate density of synapses in the cortex of the brain (8).

The immune system is actually hard wired to the nervous system via neuroimmune synapses. The most profound manifestation of this is innate immunity signals to the brain via cytokines, such as IL-1, in the febrile response, which is marked by temperature elevation accompanied by effects on appetite and arousal, mani- 


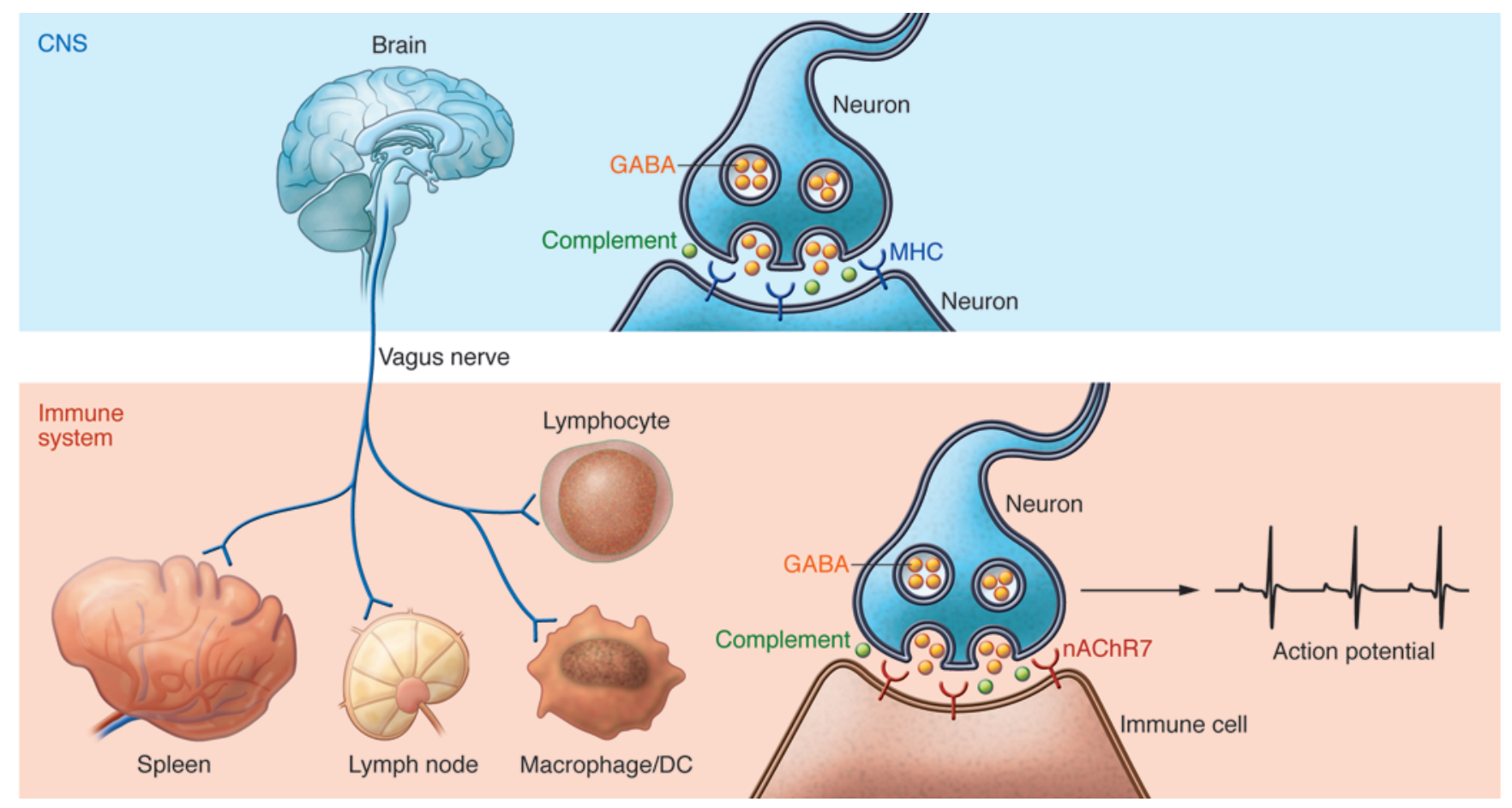

\section{Figure 1}

Shared concepts of the immune and nervous systems. The immune and nervous systems are both physically linked, as demonstrated by innervation of the spleen and other lymphoid organs, and share a common construction. Many signaling entities, such as the MHC molecules, complement, and GABA are active in both systems, and the similar physical makeup of immune and neurological synapses suggests that they both derive from a shared ancestral cellular connection.

fested by anorexia and somnolence. The nervous system is wired to immune cells in the reticuloendothelial system. Blockade of cholinergic signals via the vagus nerve can avert septic shock in animal models. There is a hard-wired cholinergic circuit involving the vagus nerve and splenic lymphoid tissue (9).

\section{Memory}

Another similarity shared between the nervous and immune systems is the concept of memory. Immunological memory is stunning: we gain lifelong immunity to many infections and immunizations that occur in childhood. Upon reexposure to an infection or to a "booster" vaccine, memory responses are detectable within hours, and $\mathrm{T}$ cells and plasmablasts are at work with life-saving rapidity to produce effective neutralizing immune responses (10). Long-term neurological memory is comparably durable to this lifelong immunological memory. However, immune memory degrades; herpes zoster can appear in one's sixth decade and after, representing a loss of immune memory of childhood varicella infection. Fortunately, we can greatly reduce the consequences of this loss of immune memory with a varicella zoster vaccination at age 60 . When neurological memory fails as we age, there are many potential causes, but the most prevalent reason is a neurodegenerative condition known as Alzheimer disease.

From two articles in this series - Czirr et al. and Ransohoff et al. $(11,12)$ - we learn that the innate immune system plays a major role in the process of neurodegeneration, seen in Alzheimer disease and other forms of dementia. Again, molecules that are well known in one system - such as complement - are also potent mediators of the formation and elimination of synapses in the other. The role of complement components in the Alzheimer brain awaits elucidation, but there is a high likelihood that complement deposits in the Alzheimer brain play a major role in the maintenance and elimination of synapses in the degenerating conditions leading to loss of memory. We should also note that well-known molecules in Alzheimer disease, such as amyloid precursor protein and its peptides, are present in damaged axons in other diseases such as multiple sclerosis, in which neurological memory is largely intact. These proteins may have Janus faces, conferring damage in one disease while playing a guardian role in another (13). One of the exciting directions in research on Alzheimer disease is the potential for a vaccine to amyloid $\beta$ (14). Just as a vaccine to varicella zoster can help the immune system recognize and fight an extrinsic pathogen, a vaccine to amyloid $\beta$ could help the body recognize and fight an endogenous pathogenic molecule (14).

\section{The immune privilege of the nervous system}

Three of the pieces in this series deal with the concept that the brain, behind the blood-brain barrier, has no classic immune system as we know it $(11,12,15)$. However, the innate and adaptive immune systems can penetrate this barrier during inflammation. There is also substantial evidence that the innate and adaptive arms of the immune system are involved in routine surveillance of the brain to guard against infection. Our experiences with HIV and with drugs that block migration of lymphocytes into the brain reveal that opportunistic infections can arise with alarming frequency if we impair the components of innate and adaptive immu- 
nity (16). Incidence of the viral encephalitis known as progressive multifocal leukoencephalopathy (PML) in patients treated for relapsing remitting multiple sclerosis with the monoclonal antibody natalizumab, an antibody that binds $\alpha 4$ integrin, is remarkable (17): over one per 400 individuals with relapsing remitting MS develop PML, when treated with natalizumab for more than two years, if they have antibodies to the John Cunningham virus, the polyoma virus causing PML. Clearly, inhibiting immune surveillance of the brain is deleterious $(16,17)$.

The adaptive immune system targets the nervous system in many diseases. In neuromyelitis optica, the immune system recognizes a water channel, AQP-4, on astrocytes. In Sydenham's chorea, a sugar on streptococcus is targeted, and the disease ensues following a group A strep infection. These antibodies recognize a cognate structure in the basal ganglia of the brain $(15,18,19)$. In an article in this series, Alyssa Nylander and David Hafler describe the numerous adaptive immune responses to components of the myelin sheath as well as to inducible molecules such as the small heat shock protein $\alpha B$-crystallin (20). Remarkably, many of the genes that predispose to MS susceptibility are those that govern both innate and adaptive immune responses, including the MHC, IL-2R, and IL-7R.

The articles in this Review series are from scientists who are working at the intersection of immunology and neuroscience, and they reveal many impressive intersections of these two systems. We can marvel at the many common design features and even the use of the same molecular components to accomplish the very diverse tasks of the brain in contrast with the immune system.

\section{Acknowledgments}

The support of the Guthy Jackson Charitable Foundation, John and Sally Endriz, and the Zimmerman Chair at Stanford University is gratefully acknowledged.

Address correspondence to: Lawrence Steinman, Stanford University School of Medicine, Department of Neurology and Neurological Sciences, Beckman Center for Molecular Medicine, 279 Campus Drive, Room B002, Stanford, California 94305, USA. Phone: 650.725.6401; Fax: 650.725.0627; E-mail: steiny@stanford.edu.
1. Irvine DJ, Purbhoo MA, Krogsgaard M, Davis MM Direct observation of ligand recognition by T cells. Nature. 2002;419(6909):845-849.

2. Shatz CJ. MHC class I: an unexpected role in neuronal plasticity. Neuron. 2009;64(1):40-45.

3. Stevens B, et al. The classical complement cascade mediates CNS synapse elimination. Cell. 2007;131(6):1164-1178.

4. Dustin ML. Signaling at neuro/immune synapses. J Clin Invest. 2012;122(4):1149-1155.

5. Clark B, Hausser M. Neural coding: hybrid ana$\log$ and digital signaling in axons. Curr Biol. 2009;16(15):R585-R588.

6. Das V, et al. Activation-induced polarized recycling targets $\mathrm{T}$ cell antigen receptors to the immunological synapse; involvement of SNARE complexes. Immunity. 2004;20(5):577-588.

7. Bhat R, et al. Inhibitory role for GABA in autoimmune inflammation. Proc Natl Acad Sci U S A. 2010;107(6):2580-2585.

8. Glynn MW, et al. MHCI negatively regulates syn- apse density during the establishment of cortical connections. Nat Neurosci. 2011;14(4):442-451.

9. Andersson U, Tracey KJ. Reflex principles of immunological homeostasis [published online ahead of print March 24, 2011]. Annu Rev Immunol. doi:10.1146/annurev-immunol-020711-075015.

10. Wrammert J, et al. Rapid cloning of high-affinity human monoclonal antibodies against influenza virus. Nature. 2008;453(7195):667-671.

11. Czirr E, Wyss-Coray T. The immunology of neurodegeneration. J Clin Invest. 2012;122(4):1156-1163.

12. Ransohoff RM, Brown MA. Innate immunity in the central nervous system. J Clin Invest. 2012; 122(4):1164-1171.

13. Wentzell JS, Bolkan BJ, Carmine-Simmen K, Swanson TL, Musashe DT, Kretzschmar D. Amyloid precursor proteins are protective in Drosophila models of progressive neurodegeneration [published online ahead of print January 10, 2012]. Neurobiol Dis. doi:http://dx.doi.org/10.1016/ j.bbr.2011.03.031.
14. Schenk D. Amyloid-beta immunotherapy for Alzheimer's disease: the end of the beginning. Nat Rev Neurosci. 2002;3(10):824-828.

15. Wraith DC, Nicholson LB. The adaptive immune system in diseases of the central nervous system. J Clin Invest. 2012;122(4):1172-1179.

16. Kaul M, Lipton SA. Mechanisms of neuroimmunity and neurodegeneration associated with HIV-1 infection and AIDS. J Neuroimmune Pharmacol. 2006;1(2):138-151.

17. Steinman L. Blocking adhesion molecules as therapy for multiple sclerosis: natalizumab. Nat Rev Drug Discov. 2005;4(6):510-519.

18. Steinman L, Merrill JT, McInnes IB, Peakman M. Optimization of current and future therapy for autoimmune diseases. Nat Med. 2012; 18(1):59-65.

19. Steinman L, Oldstone MBA. More mayhem from molecular mimics. Nat Med. 1997;3(12):1321-1322.

20. Nylander A, Hafler DA. Multiple sclerosis. J Clin Invest. 2012;122(4):1180-1188. 